\title{
Bounding by canonical functions, with $\mathrm{CH}^{*}$
}

\author{
Paul Larson ${ }^{\ddagger} \quad$ Saharon Shelah ${ }^{\S}$
}

June 9, 2021

\begin{abstract}
We show that the members of a certain class of semi-proper iterations do not add countable sets of ordinals. As a result, starting from suitable large cardinals one can obtain a model in which the Continuum Hypothesis holds and every function from $\omega_{1}$ to $\omega_{1}$ is bounded on a club by a canonical function for an ordinal less than $\omega_{2}$.
\end{abstract}

\section{Introduction}

Given an ordinal $\gamma$, a function $f: \omega_{1} \rightarrow$ Ord is a canonical function for $\gamma$ if the empty condition (i.e., $\left.\omega_{1}\right)$ in the forcing $\mathcal{P}\left(\omega_{1}\right) / N S_{\omega_{1}}$ forces that $j(f)\left(\omega_{1}^{V}\right)=\gamma$, where $j$ is the elementary embedding induced by the generic. For each $\alpha<\omega_{1}$, the constant function with value $\alpha$ is the canonical function for $\alpha$. For $\alpha \in$ $\left[\omega_{1}, \omega_{2}\right)$, a canonical function $f$ for $\alpha$ is obtained by taking a bijection $g: \omega_{1} \rightarrow \alpha$ and letting $f(\beta)$ be the ordertype of $g[\beta]$. In this paper we let Bounding denote the statement that every function from $\omega_{1}$ to $\omega_{1}$ is bounded on a club subset of $\omega_{1}$ by a canonical function for an ordinal less than $\omega_{2}$. It is fairly easy to see that if the nonstationary ideal on $\omega_{1}\left(N S_{\omega_{1}}\right)$ is saturated, then Bounding holds. The second author has shown [12] that given the existence of a Woodin cardinal there is a semi-proper forcing making $N S_{\omega_{1}}$ saturated, and it has been known for some time that there is a simpler forcing making Bounding hold from a weaker large cardinal hypothesis. The most quotable result in this paper is that this standard forcing to make every function from $\omega_{1}$ to $\omega_{1}$ bounded by a canonical function is $(\omega, \infty)$-distributive (i.e., it does not add $\omega$-sequences of ordinals), and so this statement is consistent with the Continuum Hypothesis, even in the presence of large cardinals. This is in contrast with saturation, as

\footnotetext{
*This research was conducted while both authors were in residence at the Mittag-Leffler Institute. We thank the Institute for its hospitality.

${ }^{\dagger}$ MSC 2000: 03E35, 03E50, 03E55. Keywords: Iterated Forcing, Canonical Functions, Continuum Hypothesis.

$\ddagger$ The research of the first author was supported in part by the NSERC grants of Juris Steprāns, Paul Szeptycki and Franklin D. Tall, and the Centre de Recerca Matemàtica of the Institut d'Estudis Catalan.

$\S$ The research of the second author was supported by the Israel Science Foundation, founded by the Israel Academy of Sciences. Publication number 746 .
} 
Woodin [15] has shown that if $N S_{\omega_{1}}$ is saturated and sufficiently large cardinals exist, then there is a definable counterexample to $\mathrm{CH}$. We give a more general theorem stating that the members of a certain class of semi-proper iterations are $(\omega, \infty)$-distributive. This class includes the standard forcing to make Bounding hold, and is general enough to show that a generalization of Bounding for certain sets of reals is also consistent with $\mathrm{CH}$, answering a question in [15].

The key construction used in the proof generalizes the notion of $\alpha$-properness from Chapter V of [12] to semi-proper forcing. Briefly, a forcing is $\alpha$-semi-proper if for any $\in$-chain of countable elementary submodels of length $\alpha$, there is a condition which is simultaneously semi-generic for each model in the sequence. The problem in applying the method to show that a given improper iteration is $(\omega, \infty)$-distributive is that for a given model $N$ in the sequence, $N[G] \cap \kappa$ can be a proper superset of $N \cap \kappa$, where $\kappa$ is the length of the iteration and $G$ is generic for some initial segment, so that new steps appear. For the forcings in this paper, however, we have a good understanding of how to enlarge each such $N$, as well as how to produce the appropriate tower of models to overcome this.

This can be generalized further, getting the consistency of certain forcing axioms, using ideas from [12, Chapters V and VIII, and [14. The reader is referred to [11] for more on this topic and on RCS in particular.

Interest in this question derives also from the study of Woodin's $\mathbb{P}_{\max }$ forcing 15, which produces a model in which $\mathrm{CH}$ fails and all forceable $\Pi_{2}$ sentences for $H\left(\omega_{2}\right)$ hold simultaneously. It is not known whether all such $\Pi_{2}$ sentences forceably consistent with $\mathrm{CH}$ can hold together with $\mathrm{CH}$. The generalized form of bounding in this paper is a candidate for showing that this is impossible. Candidates for the other half of the incompatibility appear in [6, 13] and in Section 10.6 of [15].

\section{Skolem Hulls}

Given a structure $M$ with a predicate $<^{*}$ for a wellordering of the domain of $M$, and a subset $X$ of the domain of $M$, we let $S k_{\left(M, \in,<^{*}\right)}(X)$ denote the Skolem hull of $X$ in $\left(M, \in,<^{*}\right)$. If $X$ is a countable elementary substructure of some $H(\chi)$ and $\eta$ is an ordinal in $X$, then we let $D_{\eta}^{X}$ be the set of all $a \in[\eta]^{<\omega}$ such that $f(a) \in X$ for all $f:[\eta]^{|a|} \rightarrow \omega_{1}$ in $X$. The point is that if $<^{*}$ is a wellordering of $H(\chi), X \prec\left(H(\chi), \in,<^{*}\right)$ is countable, and $z$ is a subset of some ordinal $\eta \in X$, then

$$
S k_{\left(H(\chi), \in,<^{*}\right)}(X \cup z)=\left\{f(a): a \in[z]^{<\omega} \wedge f \in H(\chi)^{\left([\eta]^{<\omega}\right)} \cap X\right\}
$$

and so if $z$ is such that $[z]^{<\omega} \subset D_{\eta}^{X}$,

$$
S k_{\left(H(\chi), \in,<^{*}\right)}(X \cup z) \cap \omega_{1}=X \cap \omega_{1} .
$$

Note that if $\eta<\eta^{\prime}$ are ordinals in an elementary submodel $X$, then

$$
D_{\eta}^{X}=D_{\eta^{\prime}}^{X} \cap[\eta]^{<\omega} .
$$


In order to verify that our forcings satisfy the semi-properness condition we require, we need to repeatedly apply a certain simultaneous-extendibility property. The following lemma will be useful in this regard.

Lemma 2.1. Say that $M$ is a structure and $<^{*}$ is a wellordering of the domain of $M$. Let $X$ be a countable elementary substructure of $\left(M, \in,<^{*}\right)$. Let $\eta$ be an ordinal in $X$ and let $z_{0}, z_{1}$ be countable subsets of $\eta$ such that $\left[z_{0}\right]^{<\omega} \subset D_{\eta}^{X}$ and $\left[z_{1}\right]^{<\omega} \subset D_{\eta}^{X^{\prime}}$, where $X^{\prime}=S k_{\left(M, \epsilon,<^{*}\right)}\left(X \cup z_{0}\right)$. Then $\left[z_{0} \cup z_{1}\right]^{<\omega} \subset D_{\eta}^{X}$.

Proof. Let $a \in\left[z_{0}\right]^{<\omega}$ and $b \in\left[z_{1}\right]^{<\omega}$, and let $f:[\eta]^{|a|+|b|} \rightarrow \omega_{1}$. Then $f_{a}:[\eta]^{|b|} \rightarrow \omega_{1}$, defined by letting $f_{a}(y)=f(a \cup y)$, is a function in

$$
X^{\prime}=S k_{\left(M, \in,<^{*}\right)}\left(X \cup z_{0}\right),
$$

so $f(a \cup b) \in X^{\prime} \cap \omega_{1}=X \cap \omega_{1}$.

Say that $X, Y$ are countable elementary submodels of some $\left(H(\chi), \in, \leq_{\chi}\right)$ with $X \in Y$. Let $\eta$ be an ordinal in $X$ and let $\gamma \in Y \cap \eta$. Then even though $S k_{\left(H(\chi), \in, \leq_{\chi}\right)}(X \cup\{\gamma\})$ is not directly definable in $Y$, the set itself is in $Y$, since it is equivalent to $\{f(\gamma) \mid f \in X \wedge f: \eta \rightarrow H(\chi)\}$.

2.2 Remark. Similarly, many of the arguments in this paper prove facts about sequences of elementary submodels by induction on the length of the sequence. Of course if $X, Y$ are countable elementary submodels of $\left(H(\chi), \in, \leq_{\chi}\right)$ with $X \in Y, Y$ does not see that $X$ is an elementary submodel, and so the induction hypothesis cannot be applied directly in $Y$. However, the statement about whether an object exists with a certain relation to $X$, a semi-generic extending a certain condition, say, is formalizable in $Y$, and so if one exists in $H(\chi)$ then one exists in $Y$.

\section{A class of $(\omega, \infty)$-distributive iterations}

Each step of the iterations we are considering is a forcing which shoots a continuous increasing sequence of length $\omega_{1}$ through a given stationary set of countable sets of ordinals. Under certain assumptions on the stationary set, such forcings are a typical example of improper forcings which preserve stationary subsets of $\omega_{1}$, and they have been well studied in recent years (see, for example, 3]). For the iterations in this paper, we require that these stationary sets be definable from sets from the ground model with the help of functions from $\omega_{1}$ to $\omega_{1}$ added by initial segments of the iteration.

3.1 Definition. Let $\left\langle\lambda_{\rho}: \rho<\kappa\right\rangle$ be a continuous increasing sequence of ordinals, and let $\mathcal{A}=\left\langle A_{\beta}^{\rho}: \rho<\kappa, \beta<\omega_{1}\right\rangle$ be such that each $A_{\beta}^{\rho} \subset\left[\lambda_{\rho+1}\right]^{<\omega_{1}}$. Given $\rho<\kappa$ and $f: \omega_{1} \rightarrow \omega_{1}$, let $Q_{\rho, f}$ be the forcing whose conditions are countable, continuous, increasing sequences $\left\langle x_{\beta}: \beta \leq \gamma\right\rangle$ such that for each $\beta \leq \gamma$, $x_{\beta} \cap \omega_{1} \in \omega_{1}$ and $x_{\beta} \in A_{f\left(x_{\beta} \cap \omega_{1}\right)}^{\rho}$, ordered by extension. Then an $\mathcal{A}$-iteration is a structure $\bar{Q}=\left\langle P_{\rho},{\underset{\sim}{\rho}}_{\rho}{\underset{\sim}{f} \rho}_{\rho}: \rho<\kappa\right\rangle$ such that 
(a) $\left\langle P_{\rho}, Q_{\rho}: \rho<\kappa\right\rangle$ is a Revised Countable Support iteration,

(b) each $\underset{\sim}{f_{\rho}}$ is a $P_{\rho}$-name for a function from $\omega_{1}$ to $\omega_{1}$,

(c) for all $\rho<\kappa, 1_{P_{\rho}} \Vdash Q_{\rho}=Q_{\rho, f_{\rho}^{f}}$.

The stationary sets in our iterations must also satisfy certain extendibility conditions with respect to the countable elementary submodels of a sufficiently large initial segment of the universe.

Theorem 3.2. Let $\left\langle\lambda_{\rho}: \rho\langle\kappa\rangle\right.$ be a continuous increasing sequence of strong limit cardinals with supremum $\kappa$. Fix a regular cardinal $\chi>\left(2^{\kappa}\right)^{+}$, and let $\leq_{\chi}$ be a wellordering of $H(\chi)$. Let $\mathcal{A}=\left\langle A_{\beta}^{\rho}: \rho<\kappa, \beta<\omega_{1}\right\rangle$ be such that each $A_{\beta}^{\rho} \subset\left[\lambda_{\rho+1}\right]^{<\omega_{1}}$, and let $\bar{Q}=\left\langle P_{\rho}, Q_{\rho},{\underset{\sim}{\rho}}_{\rho}: \rho<\kappa\right\rangle$ be an $\mathcal{A}$-iteration, such that the following hold.

1. For all $\rho<\kappa, \beta<\omega_{1}$, if $E \in A_{\beta}^{\rho}$ and $E^{\prime} \in\left[\lambda_{\rho+1}\right]^{<\omega_{1}}$ with $E \subset E^{\prime}$ then $E^{\prime} \in A_{\beta}^{\rho}$,

2. For all $\rho<\kappa, 1_{P_{\rho}}$ forces that for all countable $X \prec\left(H(\chi)^{V^{P_{\rho}}}, \in, \leq_{\chi}\right)$ with $\lambda_{\rho+1}, \bar{Q} \in X$, and for all $\beta<\omega_{1}$ there exists a countable $z \subset \lambda_{\rho+1}$ such that $[z]^{<\omega}$ is a subset of

$$
\bigcap\left\{D_{\lambda_{\rho+1}}^{Z}: Z \prec\left(H(\chi)^{V^{P_{\rho}}}, \in, \leq_{\chi}\right) \wedge \lambda_{\rho+1}, \bar{Q} \in Z \in X\right\}
$$

and, letting $Y=S k_{\left(H(\chi)^{\left.V^{P_{\rho}}, \in, \leq_{\chi}\right)}\right.}(X \cup z)$,

(i) $X \cap \omega_{1}=Y \cap \omega_{1}$,

(ii) $Y \cap \lambda_{\rho+1} \in A_{\beta}^{\rho}$.

3. For all countable $X \prec\left(H(\chi), \in, \leq_{\chi}\right)$ with $\bar{Q} \in X$, and for all $\rho \in X \cap$ $\kappa, \beta<\omega_{1}$ there exists a countable $Y \prec\left(H(\chi), \in, \leq_{\chi}\right)$ such that

(i) $X \subset Y$

(ii) $X \cap V_{\lambda_{\rho}+2}=Y \cap V_{\lambda_{\rho}+2}$,

(iii) $Y \cap \lambda_{\rho+1} \in A_{\beta}^{\rho}$.

Let $P$ be the Revised Countable Support limit of $\left\langle P_{\rho}, Q_{\rho}: \rho\langle\kappa\rangle\right.$. Then $P$ is $(\omega, \infty)$-distributive.

3.3 Remark. By standard RCS arguments, if in the statement of Theorem 3.2 we assume in addition that $\kappa$ is strongly inaccessible, then $P$ is $\kappa$-c.c. (see Theorem 4.6).

Conditions 2 and 3 of Theorem 3.2 could easily be subsumed into one condition; indeed, in our applications we verify both conditions at the same time. The conditions correspond to separate parts of the proof, however. Condition 2 is needed to show that each successor step of the iteration is $\alpha$-semi-proper for 
all countable $\alpha$ (Theorem 4.13), and could in fact be replaced by this requirement, though this would mean more work in applying the theorem. Condition 3 is used to construct the systems of models which we use to show that no countable sets of ordinals are added (Lemma 4.20).

Since in the end we show that $P$ does not add $\omega$-sequences, these are actually Countable Support iterations. Nonetheless, at this time we do not have a proof of (the corresponding version of) Theorem 3.2 which avoids RCS.

For the rest of this paper, sets denoted by $\kappa,\left\langle\lambda_{\rho}: \rho<\kappa\right\rangle, \chi, \leq_{\chi}, Q_{\rho, f}, \bar{Q}$, $P, \mathcal{A}$ and $\left\langle A_{\beta}^{\rho}: \rho<\kappa, \beta<\omega_{1}\right\rangle$ are supposed to have the properties given in the hypotheses of Theorem 3.2 . This policy will be modified in two ways. First, in the application sections, we will add extra properties for these terms. On the other hand, in proving the main theorem we sometimes state our lemmas more generally in terms of certain properties of these objects, temporarily forgetting the others. We hope that it is clear when we are doing this, so that there will be no confusion.

\section{Proof of Theorem 3.2}

The proof of Theorem 3.2 breaks into two largely disjoint parts, which come together only in Lemma 4.21. In the first part, we define $\alpha$-semi-proper forcing, where $\alpha$ is a countable ordinal, and show that for each countable ordinal $\alpha$, $\alpha$-semi-properness is preserved under RCS iterations. In Theorem 4.13 we use Condition 2 from Theorem 3.2 to show that each successor step from one of these iterations is forced to be $\alpha$-semi-proper for all countable $\alpha$, which then carries over to the entire iteration. In the second part of the proof we use the end-extension property given in Condition 3 of Theorem 3.2 to find a system of elementary submodels suitable for proving $(\omega, \infty)$-distributivity, which is shown in Lemmas 4.17 4.18, 4.20 and 4.21, By Theorem 4.7 the iteration is $\kappa$-c.c.

\subsection{Revised Countable Support}

The reader is referred to [12, 11] for the definition of Revised Countable Support and its basic analysis. Alternate presentations of RCS can be found in [2, 8]. We will show that the arguments here follow in all three versions. The only facts about RCS that we need outside of this section are Theorems 4.1, 4.7 and 4.11, and Property 4.3. These are (essentially - see Remark 4.4) proved in [8, 9] for the version of RCS presented in [8]. We present proofs of these facts that work for the versions of RCS in 12 and [2].

The following two theorems hold for all presentations of RCS. The first gives an important property of RCS which distinguishes it from Countable Support, that names for conditions are essentially conditions themselves.

Theorem 4.1. Say that $\left\langle P_{\alpha}, Q_{\alpha}: \alpha<\kappa\right\rangle$ is an $R C S$ iteration with $R C S$ limit $P$. Fix $p \in P$ and $\gamma<\kappa$. Suppose that $A$ is a maximal antichain in $P_{\gamma}$ below $p\lceil\gamma$ and $f: A \rightarrow P$ is a function such that for each $a \in A f(a) \leq p$ and 
$f(a)\left\lceil\gamma=a\right.$. Then there is a condition $p^{\prime} \leq p$ such that $p^{\prime} \leq p, p\left\lceil\gamma=p^{\prime}\lceil\gamma\right.$ and each $a \in A$ forces that $p^{\prime} \uparrow[\gamma, \kappa)=f(a) \uparrow[\gamma, \kappa)$.

Theorem 4.2 follows from Theorem 4.1 and Corollary 2.8 from Chapter X of [12] (and the corresponding theorem from [2]).

Theorem 4.2. Suppose that $\left\langle P_{\gamma}, Q_{\gamma}: \gamma\langle\kappa\rangle\right.$ is an RCS iteration with $R C S$ limit $P_{\kappa}$ such that each $Q_{\gamma}$ is forced to be semi-proper and each $Q_{\gamma}$ forces the corresponding $P_{\gamma}$ to have cardinality $\aleph_{1}$. Then the following hold.

1. $P_{\kappa}$ is semi-proper.

2. For all regular cardinals $\chi>2^{\left|P_{\kappa}\right|}$, for all countable $X \prec H(\chi)$ with $P_{\kappa} \in X$, for all $\gamma<\delta$ in $X \cap(\kappa+1)$, if

(a) $p \in P_{\kappa}$,

(b) $q$ is an $\left(X, P_{\gamma}\right)$-semi-generic condition in $P_{\gamma}$ below $p\lceil\gamma$,

(c) q forces "there exists $r$ in $P_{\kappa} \cap X$ such that $p \uparrow[\gamma, \kappa)=r \uparrow[\gamma, \kappa)$,"

then there exists an $\left(X, P_{\delta}\right)$-semi-generic condition $q^{\prime} \in P_{\delta}$ such that $q^{\prime}\left\lceil\gamma=q\right.$ and $q^{\prime} \leq p\lceil\delta$.

In 2], RCS is characterized by the following property.

4.3 Property. If $\left\langle P_{\alpha}: \alpha<\kappa\right\rangle$ is an RCS iteration with RCS limit $P$ and $p \in P$, then for all $q \leq p$ in $P$ there exist $\gamma<\kappa, r \in P_{\gamma}$ such that $r \leq q\lceil\gamma$ and either $r \Vdash \operatorname{cof}(\check{\kappa})=\omega$ or $r \Vdash \forall \alpha \in(\check{\gamma}, \check{\kappa}) p(\alpha)=1_{Q_{\alpha}}$.

If $\left\langle P_{\alpha}: \alpha<\kappa\right\rangle$ is an RCS iteration with RCS limit $P$ then for each condition $p$ in $P$ there is an associated $P$-name $\operatorname{supp}(p)$ for the support of $p$, the set of $\alpha<\kappa$ such that

$$
p(\alpha) \neq 1_{Q_{\alpha}},
$$

as decided by the $P$-generic filter. One can easily prove by induction on $\kappa$ that Property 4.3 implies that for each $p$ in $P \operatorname{supp}(p)$ is forced to be countable.

4.4 Remark. Property 4.3 is shown in 8 for the version of RCS in 8 , for the special case where $\kappa=\omega_{1}$ (so the first possibility for $r$ cannot hold), en route to (essentially) proving Theorem 4.6. Together these two facts give Property 4.3 for this version of RCS, since in the remaining case (where $\left|P_{\alpha}\right|<\operatorname{cof}(\kappa)$ for all $\alpha<$ $\kappa)$ a maximal antichain deciding the supremum of $\operatorname{supp}(p)$ for some condition $p$ must have maximal restriction in some initial segement of the iteration.

In [12, we have the following property (see Definition 1.1 and Claim 1.3 (1) of [12, Chapter X).

4.5 Property. If $\left\langle P_{\alpha}: \alpha<\kappa\right\rangle$ is an RCS iteration of limit length with RCS limit $P$ and $p \in P$, then there exist $P$-names $\tau_{i}(i<\omega)$ for ordinals less than $\kappa$ such that

$$
1_{P} \Vdash \operatorname{supp}(\check{p})=\left\{\tau_{i}: i<\omega\right\}
$$

and such that for each $i<\omega$ and each $q \leq p$ in $P$ there exist $\gamma<\kappa, r \in P_{\gamma+1}$ such that $r \leq q \uparrow(\gamma+1)$ and $r \Vdash \tau_{i}=\check{\gamma}$. 
If we assume that in addition each $Q_{\alpha}$ forces that the cardinality of the corresponding $P_{\alpha}$ is $\aleph_{1}$, then Property 4.5 implies Property 4.3 . We need only check the limit case. Fix $p$ and $q$, let $\left\{\tau_{i}: i<\omega\right\}$ be the sequence given by Property 4.5 with respect to $p$, and assume that there is no pair $\gamma, r$ such that

- $\gamma<\kappa$,

- $r \in P_{\gamma}$,

- $r \leq q \mid \gamma$,

- $r \Vdash \operatorname{cof}(\check{\kappa})=\omega$.

If some $P_{\alpha}$ has cardinality greater than the cofinality of $\kappa$, then (assuming that $q$ is in the generic filter), $\kappa$ will have cofinality $\omega_{1}$ in the extension by $P_{\alpha+1}$. Then, working in the $P_{\alpha+1}$-extension, let $X$ be a countable elementary substructure of a large enough $H(\chi)$ with $P, p, q,\left\{\tau_{i}: i<\omega\right\} \in X$. Define $\zeta=\bigcup(X \cap \kappa)$ and let $q^{\prime} \leq q \uparrow[\alpha+1, \kappa)$ be $\left(X, P / P_{\alpha+1}\right)$-semi-generic. Then $q^{\prime}$ forces that $\operatorname{supp}(p)$ will be contained in $\zeta$. This means that for each $i<\omega$ every condition $q^{\prime \prime} \leq q^{\prime}$ in $P / P_{\alpha+1}$ will be compatible with an $r \in P_{\zeta} / P_{\alpha+1}$ forcing that $\tau_{i}<\zeta$. But then $q^{\prime}\left\lceil\zeta\right.$ will also have this property, and thus $q^{\prime}\lceil\zeta$ also forces that $\operatorname{supp}(p) \subset \zeta$. Therefore, there exists a condition $r \in P_{\gamma}$ for some $\gamma<\kappa$ such that $r \uparrow(\alpha+1)$ forces that $\gamma$ will have the properties of $\zeta$ as above, and that $r \uparrow[\alpha+1, \gamma)$ will satisfy the properties of $q^{\prime} \uparrow \zeta$. Such an $r$ suffices.

On the other hand, if $\operatorname{cof}(k)>\left|P_{\alpha}\right|$ for all $\alpha$, then there is a sequence of pairs $\left(\gamma_{i}, r_{i}\right)(i<\omega)$ such that

- the $\gamma_{i}$ 's are increasing,

- each $r_{i} \in P_{\gamma_{i}}$ forces a bound below $\kappa$ on $\tau_{i}$,

- for all $i<j, r_{j} \mid \gamma_{i}=r_{i}$,

- each $r_{i} \leq p\left\lceil\gamma_{i}\right.$.

Then the limit of the $r_{i}$ 's is the desired condition. To find $\gamma_{i+1}, r_{i+1}$, find a maximimal antichain $A$ in $P_{\gamma_{i}}$ below $r_{i}$ such that for each $a \in A$ there is a $\gamma_{a}<\kappa$ and an $r_{a} \in P_{\gamma_{a}}$ such that

- $r_{a} \mid \gamma_{i}=a$,

- $r_{a} \leq p$,

- $r_{a} \Vdash \tau_{i}=\check{\gamma}_{a}$.

Then apply Theorem 4.1 to $A$ and the function $a \mapsto r_{a}$ to find $r_{i+1}$, and let $\gamma_{i+1}=\sup \left\{\gamma_{a}: a \in A\right\}$, which must be below $\kappa$ since $\operatorname{cof}(\kappa)>\left|P_{\gamma_{i}}\right|$.

Similar considerations give the following (see also Lemma 36.5 of [5]). The point is that the RCS limit of an iteration of cofinality $\omega_{1}$ is just the direct limit, and the ordinals of cofinality $\omega_{1}$ are stationary below $\operatorname{cof}(\kappa)$ as below. Then for any maximal antichain $A$ in $P$ as below there is some $\gamma<\kappa$ of cofinality $\omega_{1}$ such that $A \cap P_{\gamma}$ is a maximal antichain in $P_{\gamma}$. But then $|A| \leq\left|P_{\gamma}\right|$. 
Theorem 4.6. Say that $\left\langle P_{\gamma}, Q_{\gamma}: \gamma<\kappa\right\rangle$ is an RCS iteration with RCS limit $P$ such that $\operatorname{cof}(\kappa)>\left|P_{\gamma}\right|$ for all $\gamma<\kappa$, and such that each $Q_{\gamma}$ forces the corresponding $P_{\gamma}$ to have cardinality $\aleph_{1}$. Then $P$ is cof $(\kappa)$-c.c.

Applying Theorems 4.2 and 4.6 we have reduced the proof of Theorem 3.2 to showing that each $P_{\beta}(\beta<\kappa)$ is $(\omega, \infty)$-distributive.

Theorem 4.7. Let $\left\langle P_{\beta}, Q_{\beta}: \beta<\kappa\right\rangle$ be an RCS iteration of strongly inaccessible length with RCS limit $P_{\kappa}^{\sim}$ such that

- each $1_{P_{\beta}}$ forces the corresponding ${\underset{\sim}{\beta}}_{\beta}$ to be semi-proper,

- each ${\underset{\sim}{\alpha}}_{\beta}$ makes the corresponding $P_{\beta}$ have cardinality $\aleph_{1}$,

- each $\left|P_{\beta}\right|<\kappa$,

- each $P_{\beta}$ is $(\omega, \infty)$-distributive.

Then for all $\rho<\beta \leq \kappa, P_{\beta} / P_{\rho}$ is semi-proper and $\kappa$-c.c. Therefore $P_{\kappa}$ is $(\omega, \infty)$-distributive.

\subsection{Semi-generics for sequences}

The following are generalizations of ideas from Chapters V, X and XII of [12].

4.8 Definition. Let $\alpha$ be a countable ordinal.

1. The set $S E Q_{\alpha}(\chi)$ consists of all $\bar{N}=\left\langle N_{\beta}: \beta<\alpha\right\rangle$ such that

(a) each $N_{\beta}$ is a countable elementary substructure of $\left(H(\chi), \in, \leq_{\chi}\right)$,

(b) for each $\gamma<\alpha, \bar{N}\rceil \gamma=\left\langle N_{\beta}: \beta<\gamma\right\rangle \in N_{\gamma}$.

2. Let $\bar{N} \in S E Q_{\alpha}(\chi)$ and let $P$ be a forcing construction in $N_{0}$. A condition $p \in P$ is $(\bar{N}, P)$-semi-generic if

$$
p \Vdash \check{N}_{\delta}\left[G_{P}\right] \cap \omega_{1}=\check{N}_{\delta} \cap \omega_{1}
$$

for all $\delta<\alpha$, where $G_{P}$ is a $P$-name for the generic filter. The condition $p$ is $(\bar{N} \uparrow[\gamma, \beta], P)$-semi-generic if this holds for all $\delta \in[\gamma, \beta]$.

3. A forcing construction $P$ is $\alpha$-semi-proper if for every $\bar{N} \in S E Q_{\alpha}(\chi)$ with $P \in N_{0}$ and for all $p \in P \cap N_{0}$ there is an $(\bar{N}, P)$-semi generic $q \in P$ such that $p \geq q$.

Given $\bar{N} \in S E Q_{\alpha}(\chi)$ and $P \in N_{0}$, if $G \subset P$ is a generic filter then $\bar{N}[G]$ is the sequence $\left\langle N_{\beta}[G]: \beta<\alpha\right\rangle$. Both parts of the following lemma are immediate. Note that in the second part there is no need to find a $p \in G_{P}$ which is $(\bar{N}, P)$ semi-generic.

Lemma 4.9. Let $\alpha$ be a countable ordinal and fix $\bar{N} \in S E Q_{\alpha}(\chi)$. 
1. Let $P$ be an $\alpha$-semi-proper forcing in $N_{0}$, and let $Q$ be a $P$-name in $N_{0}$ for an $\alpha$-semi-proper forcing. Let $q \in P$ be $(\bar{N}, P)$-semi-generic and let $\underset{\sim}{p}$ be a $P$-name for a condition in $\left(\bigcup \bar{N}\left[G_{P}\right]\right) \cap Q$. Then there is a $P$-name $\underset{\sim}{\sim}$ for a condition in $\underset{\sim}{Q}$ such that $1_{P} \Vdash \underset{\sim}{\sim} \leq \underset{\sim}{p}$ and $(q, \underset{\sim}{r})$ is $(\bar{N}, P * Q)$-semi-generic.

2. $1 \Vdash_{P} \check{\bar{N}}\left[G_{P}\right] \in S E Q_{\check{\alpha}}(\check{\chi})^{V\left[G_{P}\right]}$.

Lemma 4.10. Let $\alpha$ be a countable ordinal. Suppose that $P_{\kappa}$ is the RCS limit of an RCS iteration $\left\langle P_{\rho}, Q_{\rho}: \rho\langle\kappa\rangle\right.$ in a set forcing extension $V^{*}$ such that each $1_{P_{\rho}}$ forces the corresponding $Q_{\rho}$ to be $\alpha$-semi-proper, and each $Q_{\rho}$ forces $P_{\rho}$ to have cardinality $\aleph_{1}$. Then the following hold in $V^{*}$.

1. $P_{\kappa}$ is $\alpha$-semi-proper.

2. Fix $\beta \leq \gamma \leq \kappa$, and fix $\bar{N} \in S E Q_{\alpha}(\chi)$ with $P_{\kappa}$ and $\beta, \gamma \in \bigcup \bar{N}$. Let $p \in P_{\kappa}, q \in P_{\beta}$ and $\delta<\alpha$ be such that

- $\beta \in N_{\delta}$,

- $p$ is $\left(\bar{N}\left\lceil\delta, P_{\kappa}\right)\right.$-semi-generic,

- $q$ is $\left(\bar{N} \uparrow[\delta, \alpha), P_{\beta}\right)$-semi-generic,

- $p\lceil\beta \geq q$,

- $q$ forces that for some $r \in P_{\kappa} \cap N_{\delta}, p \uparrow[\beta, \kappa)=r \uparrow[\beta, \kappa)$.

Let $\delta^{*} \in[\delta, \alpha)$ be such that $\gamma \in N_{\delta^{*}}$. Then there exist $q^{*} \in P_{\gamma}$ and $p^{*} \in P_{\kappa}$ such that

- $p^{*}$ is $\left(\bar{N}\left\lceil\delta^{*}, P_{\kappa}\right)\right.$-semi-generic,

- $q^{*}$ is $\left(\bar{N} \uparrow\left[\delta^{*}, \alpha\right), P_{\gamma}\right)$-semi-generic,

- $q^{*} \leq p^{*}\lceil\gamma$,

- $p^{*} \leq p$,

- $q^{*} \uparrow \beta=q$,

- $q^{*}$ forces that for some $r \in P_{\kappa} \cap N_{\delta^{*}}, p^{*} \uparrow[\gamma, \kappa)=r \uparrow[\gamma, \kappa)$.

Proof. We prove part 2 by induction on $\alpha$, working in $V^{*}$. The first part follows immediately. We fix the notation that $G_{\rho}$ is the generic filter for $P_{\rho}$, for $\rho<\kappa$. The case $\alpha=1$ is given by Theorem 4.2. For the case where $\alpha=\alpha^{\prime}+1$, there are two subcases. If $\delta^{*}<\alpha^{\prime}$, then we may assume by the induction hypothesis that $\delta^{*}=\delta$ and that there is a $\left(\bar{N} \uparrow\left[\delta^{*}, \alpha^{\prime}\right), P_{\gamma}\right)$-semi-generic $q^{\prime} \in P_{\gamma}$ such that $q^{\prime}\left\lceil\beta=q\right.$ and $q^{\prime} \leq p\left\lceil\gamma\right.$. Then since $q$ forces that $N_{\alpha^{\prime}}\left[G_{\beta}\right]$ will be elementary in $H(\chi)^{V^{*}\left[G_{\beta}\right]}$, where $G_{\beta}$ is the generic filter for $P_{\beta}$, we can replace $q^{\prime}$ with a $q^{\prime \prime}$ with the additional property that $q$ forces that $q^{\prime \prime} \uparrow[\beta, \gamma)$ will be equal to $r \uparrow[\beta, \gamma)$ for some $r \in N_{\alpha^{\prime}} \cap P_{\kappa}$. By part 2 of Theorem 4.2, then, there is a $q^{*} \in P_{\gamma}$ such that $q^{*} \uparrow \beta=q, q^{*} \leq q^{\prime \prime}$ and $q^{*}$ is $\left(N_{\alpha^{\prime}}, P_{\gamma}\right)$-semi-generic. Such a $q^{*}$ suffices.

For the subcase $\delta^{*}=\alpha^{\prime}$, by the induction hypothesis there is a $\left(\bar{N}\left\lceil\alpha^{\prime}, P_{\kappa}\right)\right.$ semi-generic $q^{\prime} \in P_{\gamma}$ such that $q^{\prime} \uparrow \beta=q$ and $q^{\prime} \leq p$. Then since $q$ forces that 
$N_{\alpha^{\prime}}\left[G_{\beta}\right]$ will be elementary in $H(\chi)^{V^{*}\left[G_{\beta}\right]}$, where $G_{\beta}$ is the generic filter for $P_{\beta}$, we can replace $q^{\prime}$ with a $q^{\prime \prime}$ with the additional property that $q$ forces that $q^{\prime \prime} \uparrow[\beta, \kappa)$ will be equal to $r \uparrow[\beta, \kappa)$ for some $r \in N_{\alpha^{\prime}} \cap P_{\kappa}$, and we can let $p^{*}=q^{\prime \prime}$. Then as in the previous paragraph, by part 2 of Theorem 4.2 there is a $q^{*}$ as desired.

The case where $\alpha$ is a limit and $\kappa=\kappa^{\prime}+1$ is similar, now fixing $\alpha$ and inducting on $\kappa$. By the induction hypothesis for $\alpha$ we may assume that $\delta=\delta^{*}$ and by the induction hypothesis for $\kappa$ we may assume that there is a $\left(\bar{N} \uparrow\left[\delta^{*}, \alpha\right), P_{\kappa^{\prime}}\right)$ semi-generic $q^{\prime} \in P_{\kappa^{\prime}}$ such that $q^{\prime} \uparrow \beta=q$ and $q^{\prime} \leq p\left\lceil\kappa^{\prime}\right.$. Since $Q_{\kappa^{\prime}}$ is forced to be $\alpha$-semi-proper, by Theorem 4.1 (or part 1 of Lemma 4.9) there is a $\left(\bar{N}, P_{\kappa}\right)$ semi-generic $q^{*}$ below $p$ such that $q^{*}\left\lceil\kappa=q^{\prime}\right.$.

For the case where $\alpha$ and $\kappa$ are both limits there are two subcases (in each of which we may assume that $\left.\delta=\delta^{*}\right)$. If $\kappa$ has cofinality $\omega$ or $\omega_{1}$, or if $\left|P_{\rho}\right|<\operatorname{cof}(\kappa)$ for each $\rho<\kappa$, then we fix an increasing sequence $\left\langle\xi_{i}: i<\omega\right\rangle$ cofinal in $\alpha$, with $\xi_{0}=\delta$. If the cofinality of $\kappa$ is countable, fix an increasing sequence of ordinals $\left\langle\beta_{i}: i\langle\omega\rangle \in N_{0}\right.$ cofinal in $\kappa$, with $\beta_{0}=\beta$. Otherwise, let $\beta_{i}$ be any ordinal in $N_{\xi_{i}}$ greater than $\sup \left(\bigcup \bar{N} \mid \xi_{i} \cap \kappa\right)$, again with $\beta_{0}=\beta$. Let $p_{0}=p$ and let $q_{0}=q$. Alternately choose conditions $p_{i+1}, q_{i+1}(i<\omega)$, such that

1. each $p_{i+1}$ is a condition in $P_{\kappa}$,

2. each $q_{i+1}$ is a condition in $P_{\beta_{i+1}}$,

3. each $q_{i} \leq p_{i}\left\lceil\beta_{i}\right.$,

4. each $p_{i+1} \leq p_{i}$,

5. for all $i<j<\omega, q_{j}\left\lceil\beta_{i}=q_{i}\right.$,

6. each $p_{i+1}$ is $\left(\bar{N}\left\lceil\xi_{i+1}, P_{\kappa}\right)\right.$-semi-generic,

7. each $q_{i+1}$ is $\left(\bar{N} \uparrow\left[\xi_{i+1}, \alpha\right), P_{\beta_{i+1}}\right)$-semi-generic,

8. each $q_{i}$ forces that for some condition $r \in P_{\kappa} \cap N_{\xi_{i}}, r \uparrow\left[\beta_{i}, \kappa\right)=p_{i} \uparrow\left[\beta_{i}, \kappa\right)$.

For the case where no condition in any $P_{\eta}$ makes $\operatorname{cof}(\kappa) \leq \omega_{1}$, we modify condition 8 as follows:

8a. each $q_{i}$ forces that for some condition $r \in P_{\kappa} \cap N_{\xi_{i}}$ such that for some $\gamma<\kappa 1_{P_{\kappa}} \Vdash \operatorname{supp}(\check{r}) \subset \check{\gamma}, r \uparrow\left[\beta_{i}, \kappa\right)=p_{i}\left[\beta_{i}, \kappa\right)$.

That such conditions exist is immediate by the induction hypothesis ( $8 \mathrm{a}$ follows from Property 4.3). Then the limit of the $q_{i}$ 's (call it $\left.q^{*}\right)$ will be the desired $\left(\bar{N}, P_{\kappa}\right)$-semi-generic, as long as it is below each $p_{i}$. This fact follows from the fact that $q^{*}$ forces that $\left\{\beta_{i}: i<\omega\right\}$ will be cofinal in $\bigcup\left\{\operatorname{supp}\left(p_{i}\right): i<\omega\right\}$. This is clear if $\operatorname{cof}(\kappa)=\omega$, and if $\operatorname{cof}(\kappa)=\omega_{1}$ it follows from the fact that each $N_{\xi_{i}} \cap \kappa$ will be cofinal in $N_{\xi_{i}}\left[G_{\beta_{i}}\right] \cap \kappa$ since $q_{i}$ is $\left(N_{\xi_{i}}, P_{\beta_{i}}\right)$-semi-generic. For the remaining case it follows from condition 8 a.

If $\left|P_{\rho}\right| \geq \operatorname{cof}(\kappa)$ for some $\rho<\kappa$, then we may assume that $\beta>\rho$ and so $\operatorname{cof}(\kappa) \leq \omega_{1}$ in the $P_{\beta}$-extension. Then we may apply the previous argument in the $P_{\beta}$-extension, along with Theorem 4.1 
Theorem 4.11. Let $\alpha$ be a countable ordinal. Say that $P_{\kappa}$ is the RCS limit of an $R C S$ iteration $\left\langle P_{\beta}, Q_{\beta}: \beta<\kappa\right\rangle$ such that each $P_{\beta+1}=P_{\beta} * Q_{\beta}$ forces $\left|P_{\beta}\right| \leq \aleph_{1}$ and each $1_{P_{\beta}}$ forces that $Q_{\beta}$ is $\alpha$-semi-proper. Then for all $\beta<\gamma \leq \kappa, P_{\gamma} / P_{\beta}$ is $\alpha$-semi-proper for every $\alpha<\omega_{1}$.

To show that each $Q_{\rho, f}$ is $\alpha$-semi-proper for all $\alpha<\omega_{1}$, we show that we can extend sequences of models in a suitable way.

Lemma 4.12. Assume that $\lambda<\chi, \bar{Q}$, and $\left\langle A_{\beta}: \beta<\omega_{1}\right\rangle \subset[\lambda]^{<\omega_{1}}$ are such that

- for all $\beta<\omega_{1}, E \in A_{\beta}$, if $E \subset E^{\prime}$ then $E^{\prime} \in A_{\beta}$,

- for all countable $X \prec\left(H(\chi), \in, \leq_{\chi}\right)$ with $\lambda, \bar{Q} \in X$, and for all $\beta<\omega_{1}$ there exists a countable $z \subset \lambda$ such that $[z]^{<\omega}$ is a subset of

$$
\bigcap\left\{D_{\lambda}^{Z}: Z \prec\left(H(\chi), \in, \leq_{\chi}\right) \wedge \bar{Q}, \lambda \in Z \in X\right\}
$$

and, letting $Y=S k_{\left(H(\chi), \epsilon, \leq_{\chi}\right)}(X \cup z)$,

(i) $X \cap \omega_{1}=Y \cap \omega_{1}$,

(ii) $Y \cap \lambda \in A_{\beta}$.

Let $\bar{N} \in S E Q_{\xi}(\chi)$ (for some $\xi<\omega_{1}$ ) with $\bar{Q}, \lambda \in N_{0}$, and let $g: \omega_{1} \rightarrow \omega_{1}$ be a function such that for each $\eta<\xi g \uparrow\left(\cup\left\{N_{\eta^{\prime}} \cap \omega_{1}: \eta^{\prime}<\eta\right\}+1\right) \in N_{\eta}$. Then there exists $\bar{N}^{\prime} \in S E Q_{\xi}(\chi)$ such that

(a) each $N_{\eta} \subset N_{\eta}^{\prime}$,

(b) each $N_{\eta}^{\prime} \cap \omega_{1}=N_{\eta} \cap \omega_{1}$,

(c) each $N_{\eta}^{\prime} \cap \lambda \in A_{g\left(N_{\eta}^{\prime} \cap \omega_{1}\right)}$,

(d) letting $N^{\prime}=\bigcup\left\{N_{\eta}^{\prime}: \eta<\xi\right\}, N^{\prime} \cap \lambda \in A_{g\left(N^{\prime} \cap \omega_{1}\right)}$.

Proof. By induction on $\xi$. If $\bar{N}$ has a last model, then we are done by the extension assumption on countable elementary submodels of $H(\chi)$. For the limit case, applying the induction hypothesis we may assume that every condition already holds, except part (d). Let

$$
N=\bigcup\left\{N_{\eta}: \eta<\xi\right\} .
$$

Applying the end-extension assumption, let $z$ be a countable subset of $\lambda$ such that

$$
[z]^{<\omega} \subset \bigcap\left\{D_{\lambda}^{Z}: Z \prec\left(H(\chi), \in, \leq_{\chi}\right) \wedge \lambda, \bar{Q} \in Z \in N\right\}
$$

and such that, letting $N^{\prime}=S k_{\left(H(\chi), \in, \leq_{\chi}\right)}(N \cup z)$, we have

1. $N \subset N^{\prime}$, 


$$
\begin{aligned}
& \text { 2. } N \cap \omega_{1}=N^{\prime} \cap \omega_{1}, \\
& \text { 3. } N^{\prime} \cap \lambda \in A_{g\left(N^{\prime} \cap \omega_{1}\right)} .
\end{aligned}
$$

Let $h: \omega \rightarrow z$ be a bijection, and fix an increasing sequence $\left\langle\zeta_{i}: i\langle\omega\rangle\right.$ cofinal in $\xi$ with $\zeta_{0}=0$. Now let each

$$
N_{\eta}^{\prime}=S k_{\left(H(\chi), \in, \leq_{\chi}\right)}\left(N_{\eta} \cup h\left[i_{\eta}\right]\right),
$$

where $i_{\eta}$ is the largest $i$ such that $\zeta_{i} \leq \eta$. Since these are all finite extensions, each initial sequence of the new sequence is an element of the later models. By the choice of $z$, each $N_{\eta} \cap \omega_{1}=N_{\eta}^{\prime} \cap \omega_{1}$, and so $\bar{N}^{\prime}$ is as desired.

Theorem 4.13. If $P_{\rho}$ is as in Theorem 3.2 and $f: \omega_{1} \rightarrow \omega_{1}$ is a function added by $P_{\rho}$, then $Q_{\rho, f}$ is $\alpha$-semi-proper in the extension by $P_{\rho}$ for all $\alpha<\omega_{1}$.

Proof. By induction on $\alpha$, using the Lemma 4.12 and working in the extension by $P_{\rho}$. Fix $\bar{N} \in S E Q_{\alpha}(\chi)$ and $p \in Q_{\rho, f}$ with $p, \bar{Q}, \lambda \in N_{0}$, and let $\bar{N}^{\prime}$ be as in Lemma 4.12, with respect to $\left\langle A_{\beta}^{\rho}: \beta<\omega_{1}\right\rangle$. If $\bar{N}^{\prime}$ has a final model (i.e., if $\alpha$ is a successor), then we can choose a $\left(\bar{N}^{\prime} \uparrow(\alpha-1), Q_{\rho, f}\right)$-semi-generic $p^{\prime} \in N_{\alpha-1}^{\prime}$ extending $p$ by the induction hypothesis (see Remark 2.2). Then since

$$
N_{\alpha-1}^{\prime} \cap \lambda_{\rho+1} \in A_{f\left(N_{\alpha-1}^{\prime} \cap \omega_{1}\right)}^{\rho},
$$

any $N_{\alpha-1}^{\prime}$-generic for $Q_{\rho, f}$ extending $p^{\prime}$ will suffice as the desired condition. For the case where $\alpha$ is a limit, we let $\bar{N}$ and $\bar{N}^{\prime}$ be as in the proof of Lemma 4.12, and fix an increasing sequence $\xi_{i}(i<\omega)$ of ordinals cofinal in $\alpha$. Then letting $p$ be $p_{0}$ we can successively pick conditions $p_{i}(i<\omega)$ such that each $p_{i+1}$ is a $\left(\bar{N}^{\prime}\left\lceil\xi_{i+1}, Q_{\rho, f}\right)\right.$-semi-generic in $N_{\xi_{i+1}}^{\prime}$ extending $p_{i}$, and such that the last member of each $p_{i+1}$ contains $N_{\xi_{i}}^{\prime} \cap \lambda_{\rho+1}$. By Condition (d) from Lemma 4.12 the limit of the $p_{i}$ 's (adjoined by their union) will be a condition in $Q_{\rho, f}$, and it will be $\left(\bar{N}, Q_{\rho, f}\right)$-semi-generic since it extends an $\left(\bar{N} \uparrow \beta, Q_{\rho, f}\right)$-semi-generic for each $\beta<\alpha$.

\subsection{Systems of models and conditions}

To show that each initial segment $P_{\beta}(\beta<\kappa)$ is $(\omega, \infty)$-distributive, we choose a suitable pair $(M, \bar{N})$ where $M$ is a countable elementary submodel of $H(\chi)$ and $\bar{N} \in S E Q_{\text {o.t. }(M \cap \beta)}(\chi)$, and find an $\left(\bar{N}, P_{\beta}\right)$-semi-generic condition in $P_{\beta}$ which extends an $M$-generic filter. As defined below, a $(\bar{Q}, \rho, \beta)$-system is a partial construction of such an object (minus the $M$-genericity requirement), where $\rho$ is the length of the initial segment for the iteration for which the desired condition has been constructed, and $\beta$ is the target length.

4.14 Definition. Given ordinals $\rho \leq \beta \leq \kappa$, we say that $(M, \bar{N}, p, q)$ is a $(\bar{Q}, \rho, \beta)$-system if (letting $\delta=$ o.t. $(M \cap \rho)$ and $\gamma=$ o.t. $(M \cap \beta)$ ):

1. $M \prec\left(H(\chi), \in, \leq_{\chi}\right)$, 
2. $\bar{Q}, \rho, \beta$ belong to $M$,

3. $\bar{N} \in S E Q_{\gamma}(\chi)$ and $\bar{Q} \in N_{0}$,

4. $q \in P_{\rho}$ and $q$ is $\left(\bar{N} \uparrow[\delta, \gamma), P_{\rho}\right)$-semi-generic,

5. for all $\rho^{\prime} \in \rho \cap M, q\left\lceil\rho^{\prime}\right.$ is $\left(N_{\text {o.t. }\left(M \cap \rho^{\prime}\right)}, P_{\rho^{\prime}}\right)$-semi-generic,

6. for all $\eta \in M \cap \beta$,

(a) for all $\nu \in N_{o . t(M \cap \eta)} \cap \omega_{1}, M \cap \lambda_{\eta+1} \in A_{\nu}^{\eta}$,

(b) $M \cap V_{\lambda_{\eta}+2} \in N_{\text {o.t. }(M \cap \eta)}$,

7. $p \in P_{\beta}, p\left\lceil\rho \geq q\right.$ and for all $\eta \in[\rho, \beta) \cap M, p\left\lceil\eta \in N_{\text {o.t. }(M \cap \eta) \text {. }}\right.$

4.15 Remark. Note that if $\beta=\rho+1$ and $(M, \bar{N}, p, q)$ is a $(\bar{Q}, \rho, \beta)$-system, then $(M, \bar{N}, p, q)$ is also a $(\bar{Q}, \beta, \beta)$-system, as (the second part of) Condition 4 becomes vacuous in the second case, and Condition 4 in the first case completes Condition 5 in the second.

4.16 Definition. Let $M \prec\left(H(\chi), \in, \leq_{\chi}\right)$ and $\beta<\kappa$ with $P_{\beta} \in M$. A filter $g \subset P_{\beta} \cap M$ is $M$-generic if for all dense sets $D \subset P_{\beta}$ in $M$ the intersection of $g$ and $D$ is nonempty. A condition $p \in M$ is a potential-M-generic for $P_{\beta}$ if there exists a $g \subset P_{\beta} \cap M M$-generic for $P_{\beta}$ such that for all $\rho \in M \cap \beta, 1_{P_{\rho}}$ forces that if

$$
M \cap \lambda_{\rho+1} \in A_{f_{\rho}\left(M \cap \omega_{1}\right)}^{\rho}
$$

then $p(\rho)=g(\rho) \frown\langle\cup g(\rho)\rangle$, and if for all $\rho \notin M \cap \beta, p(\rho)=1_{Q_{\rho}}$.

Note that if $g \subset P_{\beta}$ is $M$-generic and $p$ is in $g$ then since $\operatorname{supp}(p)$ is a $P_{\beta}$-name for a countable subset of $\beta$ the support of $p$ as determined by $g$ is contained in $M \cap \beta$. We need to see that potential- $M$-generics exist in suitable generality.

Lemma 4.17. For any $\beta \leq \kappa$, if $(M, \bar{N}, p, \emptyset)$ is a $(\bar{Q}, 0, \beta)$-system with $p \in M$, then there is a potential-M-generic $\bar{p} \leq p$ such that $\bar{p} \mid \eta \in N_{\text {o.t. }(\eta \cap M)}$ for all $\eta \in M \cap \beta$.

Proof. Given $g \subset P \cap M M$-generic for $P_{\beta}$, one can easily build a corresponding potential- $M$-generic $p$, letting $p(\rho)$ be the empty condition when

$$
M \cap \lambda_{\rho+1} \notin A_{\sim_{\rho}\left(M \cap \omega_{1}\right)}^{\rho} .
$$

The point then is just to find an $M$-generic filter $g \subset M \cap P_{\beta}$ with $p \in g$, such that $g \cap P_{\eta} \in N_{\text {o.t. }(\eta \cap M)}$ for all $\eta \in M \cap \beta$.

Inducting primarily on $\beta \in M \cap \kappa$, and secondarily on $\gamma \in M \cap \beta$, we show that if $p^{*} \in P_{\beta} \cap M$ and $g^{*} \in N_{\text {o.t. }(M \cap \gamma)}$ is an $M$-generic filter for $P_{\gamma}$ such that $p^{*} \mid \gamma \in g^{*}$ and $g^{*} \cap P_{\gamma^{\prime}} \in N_{\text {o.t. }\left(M \cap \gamma^{\prime}\right)}$ for all $\gamma^{\prime} \in M \cap \gamma$, then there exists an 
$M$-generic $g \subset P_{\beta}$ such that $g \cap P_{\gamma}=g^{*}, p^{*} \in g$ and $g \cap P_{\eta} \in N_{\text {o.t. }(M \cap \eta)}$ for all $\eta \in M \cap \beta$. Note that for each $\gamma \in M \cap \beta$, since

$$
\mathcal{P}\left(\mathcal{P}\left(\lambda_{\gamma}\right)\right) \cap M \in N_{\text {o.t. }(M \cap \gamma)}
$$

(and $\left.\left|P_{\gamma}\right| \leq 2^{\lambda_{\gamma}}\right)$, the $M$-genericity of $g^{*}$ can be verified in $N_{\text {o.t. }(M \cap \gamma)}(M$ itself is not in any member of $\bar{N}$, so this is not automatic).

The base case $\beta=0$ is trivial. For the successor step, we may assume that $\gamma+1=\beta$ Then since for the successor case there is no restriction on the last coordinate of the $M$-generic filter (other than $M$-genericity), $g^{*}$ can be extended in any fashion to an $M$-generic for $P_{\beta}$.

For the limit, fix an increasing sequence $\eta_{i}(i<\omega)$ cofinal in $\beta \cap M$, and let $p=p_{0}$. Now in $\omega$ many steps alternately pick

- $M$-generic $g_{i} \subset P_{\eta_{i}}$ in $N_{\text {o.t. }\left(M \cap \eta_{i}\right)}$ (applying the induction hypothesis plus the elementarity of $N_{\text {o.t. }\left(M \cap \eta_{i}\right)}$ plus the fact that $\bar{N}\left\lceil\right.$ o.t. $\left(M \cap \eta_{i}\right) \in$ $N_{\text {o.t. }\left(M \cap \eta_{i}\right)}$, as in Remark 2.2) with $p_{i}\left\lceil\eta_{i} \in g_{i}\right.$ such that

- $g_{i} \cap P_{\eta} \in N_{\text {o.t. }(M \cap \eta)}$ for all $\eta \in M \cap \eta_{i}$,

$-g_{i} \cap P_{\eta_{j}}=g_{j}$ for all $j<i$,

- $p_{i+1} \leq p_{i}$ in $M$ meeting the $i$ th dense set in $M$ for $P_{\beta}$ such that $p_{i+1}\left\lceil\eta_{i} \in g_{i}\right.$ (such a $p_{i+1}$ exists because $g_{i}$ is $M$-generic for $P_{\eta_{i}}$ ).

Since each $p_{i+1} \in M$, its initial segments are automatically in the corresponding $N_{\xi}$ 's. Then $\left\{p_{i}: i<\omega\right\}$ generates an $M$-generic filter $g$ for $P_{\beta}$, and for each

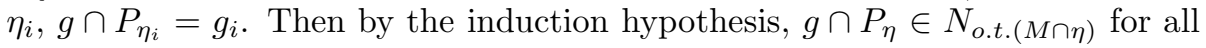
$\eta \in M \cap \beta$, since for all $i<\omega$ with $\eta_{i} \geq \eta, g \cap P_{\eta}=g_{i} \cap P_{\eta}$.

Lemma 4.18. Fix $\beta \leq \kappa$. If for all $p_{0} \in P_{\beta}$ there is a $(\bar{Q}, \beta, \beta)$-system $(M, \bar{N}, p, q)$ with $p_{0} \in M$ and $p \leq p_{0}$ a potential-M-generic, then the forcing $P_{\beta}$ is $(\omega, \infty)$-distributive.

Proof. Towards a contradiction, fix the least $\beta$ for which the lemma fails, and let $p_{0}$ be a condition in $P_{\beta}$ forcing that $P_{\beta}$ adds a new $\omega$-sequence of ordinals. Let $(M, \bar{N}, p, q)$ be a $(\bar{Q}, \beta, \beta)$-system with $p_{0} \in M$ and $p \leq p_{0}$ a potential- $M$ generic, as given by the hypothesis of the lemma. Then there exists a $P_{\beta}$-name $\tau$ in $M$ such that $p_{0}$ forces that $\tau$ will be a new $\omega$-sequence of ordinals. Let $g \subset P_{\beta} \cap M$ be an $M$-generic filter witnessing that $p$ is a potential- $M$-generic. We wish to see that $q$ is below each member of $g$. Then we will be done, as for each integer $i$ there is a member of $g$ intersecting the antichain in $P_{\beta}$ determining the $i$ th member of $\tau$. So we will show by induction on $\rho \in(\beta+1) \cap M$ that $q\left\lceil\rho \leq p^{\prime} \uparrow \rho\right.$ for all $p^{\prime} \in g$ (simultaneously). The cases where $\rho=0$ or $\rho$ is a limit and $M \cap \rho$ is cofinal in $\rho$ are clear.

For the successor step from $\rho$ to $\rho+1, f_{\rho}\left(M \cap \omega_{1}\right)$ is a $P_{\rho}$-name in $N_{\text {o.t. }(M \cap \rho)}$ for a countable ordinal, so by Conditions [5 and 6 of Definition 4.14, $q\lceil\rho$ forces that

$$
M \cap \lambda_{\rho+1} \in A_{\sim}^{\rho}{\underset{\sim}{\rho}\left(M \cap \omega_{1}\right)} .
$$


Then $q\lceil\rho$ forces that $p(\rho)=g(\rho) \frown(\cup g(\rho))$. By Condition 7 of Definition 4.14] $q \uparrow(\rho+1) \leq p \uparrow(\rho+1)$, so $q\left\lceil\rho\right.$ forces that $q(\rho)$ extends $p(\rho)$. Now fix $p^{\prime} \in g$. Since $q\left\lceil\rho\right.$ forces that $p(\rho)$ extends $p^{\prime}(\rho)$, we have that $q \uparrow(\rho+1) \leq p^{\prime} \uparrow(\rho+1)$.

Lastly, for the case where $\rho$ is a limit and $M \cap \rho$ is not cofinal in $\rho$, note that $\rho$ has uncountable cofinality, and also that we have shown that each $P_{\rho^{\prime}}$, $\rho^{\prime}<\rho$ is $(\omega, \infty)$-distributive. By Property 4.3 then, densely many conditions in $P_{\rho}$ (and therefore $g \cap P_{\rho}$ ) are conditions in some $P_{\rho^{\prime}}, \rho^{\prime}<\rho$, and so densely many conditions in $g \cap P_{\rho}$ are in some $P_{\rho^{\prime}}$ with $\rho^{\prime} \in \rho \cap M$.

Given a countable $X \prec\left(H(\chi), \in, \leq_{\chi}\right)$ with $\lambda \in X$, and given $\eta<\lambda$, we say that $Y \prec\left(H(\chi), \in, \leq_{\chi}\right)$ is a minimal $(\eta, \lambda)$-extension of $X$ if the following hold.

1. $X \cap \eta=Y \cap \eta$.

2. $Y=S k_{\left(H(\chi), \epsilon, \leq_{\chi}\right)}(X \cup A)$, for some $A \subset \lambda$.

The fact about these extensions that we will use is given in the following lemma.

Lemma 4.19. Let $X \prec\left(H(\chi), \in, \leq_{\chi}\right)$, and let $\lambda<\gamma$ be ordinals in $X$ with $\gamma$ a regular cardinal. Let $Y$ be a minimal $(\eta, \lambda)$-extension of $X$ for some $\eta<\lambda$. Then $X \cap \gamma$ is cofinal in $Y \cap \gamma$.

Proof. Since $\gamma$ is regular, each $f:[\lambda]^{<\omega} \rightarrow \gamma$ has bounded range below $\gamma$. If $f$ is in $X$ then this bound exists in $X$.

Lemma 4.20. For any set $x \in H(\chi)$ and any ordinal $\beta \leq \kappa$ there is a $(\bar{Q}, 0, \beta)$ system $\left(M, \bar{N}, 1_{P_{\beta}}, \emptyset\right)$ with $x \in M$.

Proof. Let $M_{0} \prec\left(H(\chi), \in, \leq_{\chi}\right)$ be countable with $\{\bar{Q}, \beta, x\} \in M$. For some $\zeta \leq \omega_{1}$ we build $\left\langle M_{\xi}: \xi \leq \zeta\right\rangle,\left\langle\gamma_{\xi}: \xi \leq \zeta\right\rangle$ and $\left\langle N_{\xi}: \xi<\zeta\right\rangle$ satisfying the following conditions.

1. Each $M_{\xi}$ is a countable elementary submodel of $\left(H(\chi), \in, \leq_{\chi}\right)$.

2. Each $N_{\xi}$ is a countable elementary submodel of $\left(H(\chi), \in, \leq_{\chi}\right)$.

3. Each $\gamma_{\xi}$ is the $\xi$ th ordinal in $M_{\xi}$ (0 being the 0 th ordinal).

4. $\bar{Q} \in N_{0}$.

5. For all $\xi<\zeta,\left\langle N_{\eta}: \eta<\xi\right\rangle \in N_{\xi}$.

6. For all $\xi<\zeta, M \cap V_{\lambda_{\xi}+2} \in N_{\xi}$.

7. For all $\xi<\zeta$ and for all $\nu \in N_{\xi} \cap \omega_{1}, M_{\xi+1} \cap \lambda_{\gamma_{\xi}+1} \in A_{\nu}^{\gamma_{\xi}}$.

8. For each $\xi<\zeta, M_{\xi+1}$ is a minimal $\left(2^{2^{\lambda} \gamma_{\xi}}, \lambda_{\gamma_{\xi}+1}\right)$-extension of $M_{\xi}$,

9. If $\xi$ is a limit ordinal, then $M_{\xi}=\bigcup\left\{M_{\eta}: \eta<\xi\right\}$.

10. If there exists a $\xi<\omega_{1}$ such that $\gamma_{\xi}=\beta$, then $\zeta$ is the least such $\xi$; otherwise $\zeta=\omega_{1}$. 
Given $M_{\xi}$, by applying Condition 3 in the statement of Theorem 3.2 repeatedly, once for each $A_{\nu}^{\gamma_{\xi}}$ with $\nu=N_{\xi} \cap \omega_{1}$, we can choose each $M_{\xi+1}$ to meet Conditions 7 and 8 , While the model $Y$ resulting from this repeated application may not be a minimal $\left(2^{2^{\lambda \gamma_{\xi}}}, \lambda_{\gamma_{\xi}+1}\right)$-extension of $M_{\xi}$,

$$
Y^{*}=S k_{\left(H(\chi), \epsilon, \leq_{\chi}\right)}\left(M_{\xi} \cup\left(Y \cap \lambda_{\gamma_{\xi}+1}\right)\right)
$$

will be, as $Y \cap V_{\lambda_{\gamma_{\xi}+1}}=Y^{*} \cap V_{\lambda_{\gamma_{\xi}+1}}$, so we can take this $Y^{*}$ as our $M_{\xi+1}$. The rest of the construction is straightforward.

We claim that for each $\xi \leq \zeta$ the sequence $\left\langle\gamma_{\eta}: \eta \leq \xi\right\rangle$ lists the first $\xi+1$ ordinals of $M_{\xi}$ in increasing order. This follows by induction on $\xi$. It is clear when $\xi=0$, and when $\xi=\xi^{\prime}+1$ it follows from Condition 8 and the fact that

$$
2^{2^{\lambda} \gamma_{\xi^{\prime}}}>\gamma_{\xi^{\prime}}
$$

(so in this case $\gamma_{\xi}=\gamma_{\xi^{\prime}}+1$ ). If $\xi$ is a limit ordinal, we have from the fact that $M_{\xi}=\bigcup\left\{M_{\eta}: \eta<\xi\right\}$ and the induction hypothesis that $\left\langle\gamma_{\eta}: \eta<\xi\right\rangle$ lists the first $\xi$ ordinals of $M_{\xi}$ in increasing order. Then the definition of $\gamma_{\xi}$ finishes the proof of the claim.

Next we claim that $\gamma_{\xi}=\beta$ for some $\xi<\omega_{1}$, and so $\zeta<\omega_{1}$. Given this we are done, letting $M=M_{\zeta}$ and $\bar{N}=\left\langle N_{\xi}: \xi<\zeta\right\rangle$. All the conditions of Definition 4.14 are satisfied trivially, aside from Condition [6. Condition 6] is satisfied since for each $\eta \in M_{\zeta} \cap \beta$, there is some $\xi<\zeta$ such that $\eta=\gamma_{\xi}$ (and so $\xi=$ o.t. $(M \cap \eta)$ ). Then $N_{\xi}$ and $M_{\xi}$ were chosen to satisfy Condition 6 of Definition 4.14 by Conditions 6 and 7 of the construction, and this relationship was preserved for all later $M_{\xi}$ by Condition 8 ,

Assume to the contrary that $\zeta=\omega_{1}$. If $\gamma_{\omega_{1}}=\beta$, then since every ordinal in $M_{\omega_{1}} \cap \beta$ is equal to some $\gamma_{\xi}$, there is a limit ordinal $\xi<\omega_{1}$ such that

$$
\left\{\gamma_{\eta}: \eta<\xi\right\}=M_{\xi} \cap \beta .
$$

But then $\gamma_{\xi}=\beta$, contradicting $\zeta=\omega_{1}$. So we may assume that $\gamma_{\omega_{1}}<\beta$. We will show that the cofinality of $M_{\omega_{1}} \cap \gamma_{\omega_{1}}$ is countable, which is a contradiction since $\left\langle\gamma_{\xi}: \xi<\omega_{1}\right\rangle$ is increasing and cofinal in it. If $\gamma_{\omega_{1}} \leq \lambda_{\gamma_{\xi}}$ for some $\gamma_{\xi}$ with $\gamma_{\omega_{1}} \in M_{\xi}$, then $M \cap \gamma_{\omega_{1}}=M_{\xi} \cap \gamma_{\omega_{1}}$, which is countable. If not, $\gamma_{\omega_{1}}=\lambda_{\gamma_{\omega_{1}}}$. If $\gamma_{\omega_{1}}$ is singular, let $\rho$ be the cofinality of $\gamma_{\omega_{1}}$. Then $\rho<\lambda_{\gamma_{\xi}}$ for some $\gamma_{\xi}$ with $\left\{\rho, \gamma_{\omega_{1}}\right\} \in M_{\xi}$, and there exists a cofinal map $f: \rho \rightarrow \gamma_{\omega_{1}}$ in $M_{\xi}$. Since $\rho \cap M_{\omega_{1}}=\rho \cap M_{\xi}, f\left[M_{\xi} \cap \rho\right]$ is a countable set cofinal in $M_{\omega_{1}} \cap \gamma_{\omega_{1}}$. The last remaining case is that $\gamma_{\omega_{1}}$ is a regular limit cardinal. Let $\xi$ be least with $\gamma_{\omega_{1}} \in M_{\xi}$, and fix a cofinal sequence in $M_{\xi} \cap \gamma_{\omega_{1}}$. By Lemma 4.19 and Condition 8 of the construction, this sequence is cofinal in $M_{\omega_{1}} \cap \gamma_{\omega_{1}}$.

We finish by applying the following lemma to the case $\rho=0, \gamma=\beta$, from Lemma 4.20, By Lemma 4.18, then, we are done.

Lemma 4.21. Fix $\beta \leq \kappa$. Let $(M, \bar{N}, p, q)$ be a $(\bar{Q}, \rho, \beta)$-system with $\rho \leq \gamma \leq \beta$ in $M$, and assume that $p$ is a potential- $M$-generic. Then there exists a condition $q^{\prime} \in P_{\gamma}$ such that 
1. $q^{\prime} \uparrow \rho=q$,

2. $\left(M, \bar{N}, p, q^{\prime}\right)$ is a $(\bar{Q}, \gamma, \beta)$-system.

Proof. We first note that the lemma follows from the restricted version of the lemma where $\gamma=\beta$. To see this, fix $M, \bar{N}, p, q, \rho, \gamma, \beta$ as given by the hypothesis of the unrestricted version, and note that the restricted version then gives a $q^{*} \in P_{\gamma}$ such that $q^{*}\left\lceil\rho=q\right.$ and $\left(M, \bar{N}\left\lceil\right.\right.$ o.t. $(M \cap \gamma), p\left\lceil\gamma, q^{*}\right)$ is a $(\bar{Q}, \gamma, \gamma)$-system. Now, if $G_{\rho} \subset P_{\rho}$ is $V$-generic with $q \in G_{\rho}$, then $M\left[G_{\rho}\right] \cap \operatorname{Ord}=M \cap \operatorname{Ord}$ (since $p$ is a potential- $M$-generic and $q \leq p)$ and the following hold.

- $\forall \alpha<$ o.t. $(\beta \cap M) N_{\alpha}\left[G_{\rho}\right] \cap \omega_{1}=N_{\alpha} \cap \omega_{1}$,

- $\bar{N}\left[G_{\rho}\right]=\left\langle N_{\alpha}\left[G_{\rho}\right]: \alpha<\right.$ o.t. $\left.(M \cap \beta)\right\rangle \in S E Q_{\text {o.t. }(M \cap \beta)}(\chi)^{V\left[G_{\rho}\right]}$.

Furthermore, in $V\left[G_{\rho}\right]$,

- $q^{*} \uparrow[\rho, \gamma) \in P_{\gamma} / P_{\rho}$

- $\forall \gamma^{\prime} \in(\gamma \backslash \rho) \cap M, q^{*} \uparrow\left[\rho, \gamma^{\prime}\right)$ is $\left(N_{\text {o.t. }\left(M \cap \gamma^{\prime}\right)}\left[G_{\rho}\right], P_{\gamma^{\prime}} / P_{\rho}\right)$-semi-generic,

- $p \uparrow[\rho, \gamma) \geq q^{*} \uparrow[\rho, \gamma)$.

Applying the elementarity of $N_{\text {o.t. }(M \cap \gamma)}\left[G_{\rho}\right]$ in $H(\chi)^{V\left[G_{\rho}\right]}$, we see that there exists a condition $\bar{q} \in N_{\text {o.t. }(M \cap \gamma)}\left[G_{\rho}\right] \cap P_{\gamma} / P_{\rho}$ satisfying these conditions. By part 2 of Lemma 4.10, then, there is a condition $q^{\prime} \in P_{\gamma}$ such that $q^{\prime} \uparrow \rho=q$ and $q$ forces that $q^{\prime} \uparrow[\rho, \gamma)$ is a

$$
\left(\bar{N}\left[G_{\rho}\right] \uparrow[\text { o.t. }(M \cap \gamma) \text {, o.t. }(M \cap \beta)), P_{\gamma} / P_{\rho}\right) \text {-semi-generic }
$$

condition extending such a $\bar{q}$. This $q^{\prime}$ suffices. We have Conditions 5 and 7 of Definition 4.14 by the properites listed above for $q^{*} \uparrow[\rho, \gamma)$, Condition 4 by our extension, and the others by the assumptions of the lemma.

The restricted version of the lemma follows by induction on o.t. $((\beta \backslash \rho) \cap M)$. Note that our induction hypothesis entitles us (once we have fixed $\beta$ and $\rho$ ) to assume that the unrestricted version holds whenever $\gamma<\beta$.

Now, when $\rho=\beta$ there is nothing to show. The argument for increasing o.t. $((\beta \backslash \rho) \cap M)$ by one follows from the case where $\beta=\rho+1$, and this case is also trivial (see Remark 4.15).

The only remaining case then is when $\rho<\beta$ and $\beta=\gamma$ is a limit ordinal. Fix an increasing sequence $\eta_{i}(i<\omega)$ cofinal in $M \cap \beta$ with $\eta_{0}=\rho$, and succesively apply the induction hypothesis (in $V$, as opposed to some submodel). That is, let $q_{0}=q$, and given $q_{i}$, let $q_{i+1} \in P_{\eta_{i+1}}$ be such that $q_{i+1}\left\lceil\eta_{i}=q_{i}\right.$ and $\left(M, \bar{N}, p, q_{i+1}\right)$ is a $\left(\bar{Q}, \eta_{i+1}, \beta\right)$-system. Then each $q_{i}$ is $\left(\bar{N} \uparrow\left[\right.\right.$ o.t. $\left(M \cap \eta_{i}\right)$, o.t. $(M \cap$ $\beta)$ ), $P_{\eta_{i}}$ )-semi-generic, and the limit of the $q_{i}$ 's is the desired $q^{\prime}$. Condition 4. of Definition 4.14 is then easily satisfied (the second half being vacuous), and Conditions 5 and 7 being local properties, are satisfied by the induction hypothesis. 


\section{Applications}

\subsection{Bounding}

The following is a minor modification of a standard fact.

Theorem 5.1. Let $\lambda$ be a measurable cardinal, let $\theta$ be a regular cardinal such that $V_{\lambda+2} \subset H(\theta)$, and let $\leq^{*}$ be a wellordering of $H(\theta)$. Then for every countable ordinal $\beta$ and every $X \prec\left(H(\theta), \in, \leq^{*}\right)$, there is a $z \in[\lambda]^{\beta}$ such that

$$
(* *)[z]^{<\omega} \subset \bigcap\left\{D_{\lambda}^{Z}: Z \prec\left(H(\theta), \in, \leq^{*}\right) \wedge Z \in X\right\},
$$

and such that, letting

$$
Y=S k_{\left(H(\theta), \in, \leq^{*}\right)}(X \cup z),
$$

$X \cap \lambda$ is a proper initial segment of $Y \cap \lambda$.

Proof. It suffices to prove the theorem for the case $\beta=1$, since then we may repeat the construction any countable ordinal number of times. To see that $(* *)$ is preserved, note that if after repeating the construction some countable number of times we have the $\lambda$-end-extension $X^{\prime}$ of $X$ and we have added $\gamma_{0}, \ldots, \gamma_{n}$ (possibly among other ordinals) to $z$ and $Z \prec\left(H(\theta), \in, \leq^{*}\right)$ is in $X$, then $Z^{\prime}=S k_{\left(H(\theta), \in, \leq^{*}\right)}\left(Z \cup\left\{\gamma_{0}, \ldots, \gamma_{n}\right\}\right) \in X^{\prime}$. Further, if $\gamma_{n+1} \in D_{\lambda}^{Z^{\prime}}$, then by Lemma 2.1. $\left\{\gamma_{0}, \ldots, \gamma_{n+1}\right\} \in D_{\lambda}^{Z}$.

Now, to prove the theorem for the case $\beta=1$, let $\mu$ be the $\leq^{*}$-least normal measure on $\lambda$, and fix $X$ as in the statement of the theorem. Let $\gamma$ be any member of $\bigcap(X \cap \mu)$, and let

$$
Y=S k_{\left(H(\theta), \in, \leq^{*}\right)}(X \cup\{\gamma\}) .
$$

The key point is that if $f: \lambda \rightarrow \lambda$ is a regressive function in any elementary submodel of $\left(H(\theta), \in, \leq^{*}\right)$, then $f$ is constant on a set $A \in \mu$, and since $\mu$ is $\leq^{*}$-least, $\mu$ and therefore $A$ and the constant value are also in the model. Along with the fact that $\bigcap(X \cap \mu) \subset \bigcap(Z \cap \mu)$ for any $Z \prec\left(H(\theta), \in, \leq^{*}\right)$ with $Z \in X$, this shows that $\gamma$ satisfies $(* *)$. To see that $Y \cap \lambda$ is a proper end-extension of $X \cap \lambda$, note that $\gamma \notin X$, and since any ordinal $\eta \in Y \cap \lambda$ is of the form $f(\gamma)$ for some $f: \lambda \rightarrow \lambda$ in $X$, if $\eta \in Y \cap \gamma$ then any corresponding $f$ is regressive on a set in $\mu$, and so $\eta \in X$, by the key point.

Putting together Theorems 3.2 and 5.1 we have the following.

Corollary 5.2. Say that there exists a strongly inaccessible limit of measurable cardinals. Then there is a semi-proper forcing extension in which Bounding holds, along with the Continuum Hypothesis.

Proof. Let $\left\langle\lambda_{\rho}: \rho\langle\kappa\rangle\right.$ be a continuous increasing sequence of cardinals with supremum $\kappa$ strongly inaccessible such that each $\lambda_{\rho+1}$ is a measurable cardinal. Fix a regular cardinal $\chi>\left(2^{\kappa}\right)^{+}$, and let $\leq_{\chi}$ be a wellordering of $H(\chi)$. Let

$$
\mathcal{A}=\left\langle A_{\beta}^{\rho} \subset\left[\lambda_{\rho+1}\right]^{<\omega_{1}}: \rho<\kappa, \beta<\omega_{1}\right\rangle
$$


be such that each $A_{\beta}^{\rho}$ is the set of countable subsets of $\lambda_{\rho+1}$ of ordertype greater than $\beta$. Then by Theorem 5.1, Conditions 1,3 of Theorem 3.2 are satisfied (Condition 2 by the fact that small forcing preserves measurable cardinals), so any forcing $P$ as in the statement of Theorem 3.2 is semi-proper and $(\omega, \infty)$ distributive. We may further require that for every function $f$ added by some initial stage of the iteration there is a $\rho<\kappa$ such that $f=f_{\rho}$. Each $Q_{\rho, f}$ forces that $\left|\lambda_{\rho+1}\right|=\aleph_{1}$ and that any canonical function for $\lambda_{\rho+1}$ dominates $f$ on a club, so $P$ forces Bounding. Further, since $2^{\omega}<\kappa$ in $V$ and $P$ forces $\kappa=\omega_{2}, P$ forces $\mathrm{CH}$.

Deiser and Donder have recently shown [1] that Bounding is equiconsistent with a strongly inaccessible limit of measurable cardinals.

\subsection{Suslin Bounding}

The previous application can be generalized to show that the following statement can be forced without adding reals, answering a question in [15].

5.3 Definition. (4]) Suppose that $A \subset \mathbb{R}$. Then $A$ is universally Baire if for any compact Hausdorff space $X$ and any continuous function $\pi: X \rightarrow \mathbb{R}$, the set $\{a \in X \mid \pi(a) \in A\}$ has the property of Baire in $X$.

5.4 Definition. Suslin Bounding is the following statement. Suppose that $A \subset$ $\mathbb{R}$ is universally Baire, and fix a function $f: \omega_{1} \rightarrow A$. Then there is a tree $T$ on $\omega \times \omega_{1}$ such that $A=p[T]$ and such that $\left\{\alpha<\omega_{1} \mid f(\alpha) \in p[T \uparrow(\omega \times \alpha)]\right\}$ contains a club.

Theorem 5.5. Bounding is equivalent to Suslin Bounding for $\Pi_{1}^{1}$ sets.

Proof. For the forward direction, we adapt the proof that $\Pi_{1}^{1}$ sets are projections of trees on $\omega \times \omega_{1}$ (see [10]). Let $S$ be a tree on $\omega \times \omega$, let $B=p[T]$ and let $A=\omega^{\omega} \backslash B$. Recall that for all $x \in A$,

$$
S_{x}=\left\{\sigma \in \omega^{<\omega} \mid(x \Uparrow|\sigma|, \sigma) \in S\right\}
$$

is wellfounded. For each $x \in A$, let $\beta_{x}$ be the least ordinal (necessarily countable) such that there exists a function $r_{x}: \omega^{<\omega} \rightarrow \beta_{x}$ with the property that if $\sigma, \sigma^{\prime} \in$ $S_{x}$ are such that $\sigma$ is a proper initial segment of $\sigma^{\prime}$, then $r_{x}(\sigma)>r_{x}\left(\sigma^{\prime}\right)$. Fix $f: \omega_{1} \rightarrow A$, and for each $\alpha<\omega_{1}$ let $h(\alpha)=\beta_{f(\alpha)}$. Now fix $\gamma<\omega_{2}$ and a bijection $g: \omega_{1} \rightarrow \beta$ such that for every $\alpha$ in a fixed club $C \subset \omega_{1}$, o.t. $(g[\alpha])>h(\alpha)$. Let $\sigma: \omega \rightarrow \omega^{<\omega}$ be a bijection such that $|\sigma(i)| \leq i+1$ for all $i<\omega$. Let $T$ be the tree on $\omega \times \omega_{1}$ consisting of all pairs $(\rho, \tau)$ such that if $i, j<|\rho|(=|\tau|)$ and $\sigma(i)$ is a proper initial segment of $\sigma(j)$ with

$$
(\rho \uparrow|\sigma(i)|, \sigma(i)),(\rho \uparrow|\sigma(j)|, \sigma(j))
$$

both in $S$, then $g(\tau(i))>g(\tau(j))$. Then $p[T]=A$, and, for each $x \in A$, if $\delta_{x}$ is the least (again, necessarily countable) ordinal $\delta$ such that o.t. $(g[\delta]) \geq \beta_{x}$ and 
$i_{x}$ is an order preserving embedding of $\beta_{x}$ into $g\left[\delta_{x}\right]$, then $\left(x, g^{-1} \circ i_{x} \circ r_{x} \circ \sigma\right)$ is a path through $T \uparrow\left(\omega \times \delta_{x}\right)$. Since $\delta_{f(\alpha)} \leq \alpha$ for all $\alpha \in C$, this shows that $T$ satisfies the definition of Suslin Bounding for $f$.

For the other direction, let $W \subset \omega^{\omega}$ be the set of functions coding wellordings of $\omega$ under some fixed coding with the property that for each $g \in \omega^{\omega}$ and $n \in \omega$, $g \uparrow(n+1)$ codes how $n+1$ compares with each $m<n+1$. Each function from $\omega_{1}$ to $\omega_{1}$ induces a corresponding function from $\omega_{1}$ into $W$. Let $f: \omega_{1} \rightarrow W$ be such a function, and let $T$ be the tree given by Suslin Bounding, with $C$ the witnessing club set. We have a partial, not necessarily transitive, order $\leq_{o}$ on the sequences in $T$, where $\sigma_{0} \leq_{o} \sigma_{1}$ if one extends the other, and, $\sigma_{i}$ being the longer one, the first cordinate of the last element of $\sigma_{i}$ codes that $\left|\sigma_{0}\right|<\left|\sigma_{1}\right|$ in the corresponding ordering. Let $\leq_{t}$ be the least transitive ordering containing $\leq_{o}$. Then $\leq_{t}$ is a wellfounded partial order. Seeing this requries checking that the resulting order is antireflexive and wellfounded. To see antireflexivity, assume that

$$
\tau \leq_{o} \sigma_{0} \leq_{o} \ldots \leq_{o} \sigma_{n} \leq_{o} \tau
$$

is the shortest possible counterexample. First note that $\sigma_{0}$ and $\sigma_{n}$ cannot be comparable in $\leq_{o}$ (or identical) since then there would be a shorter counterexample, removing the $\tau$ 's and placing either $\sigma_{n}$ and the beginning of the sequence or $\sigma_{0}$ at the end if they are unequal. Therefore $\sigma_{0}$ and $\sigma_{n}$ must be incompatible extensions of $\tau$, and $\sigma_{1}$ must be comparable with $\tau$ and distinct from $\sigma_{n}$. But then depending on whether $\sigma_{1} \geq_{o} \tau$ or $\tau \geq_{o} \sigma_{1}$, there is a shorter counterexample, removing either $\sigma_{0}$ or $\sigma_{2} \ldots \sigma_{n}$ from the original sequence. To see wellfoundedness, let $\sigma_{i}(i<\omega)$ be a descending sequence in $\leq_{o}$. First note that if some $\tau \in T$ has infinitely many extensions in the sequence, then all but finitely many of them must be extensions of a fixed immediate successor of $\tau$, since otherwise initial segments of $\tau$ are visited infinitely often by the sequence, which is impossible (using antireflexivity of $\leq_{t}$ ), there being only finitely many of them. But the empty sequence has infinitely many extension in the sequence, which means that we can build an infinite chain though $T$ all of whose members have this property. We claim that infinitely many members of this chain must be in the sequence, which gives a contradiction since the chain codes a wellordering. To see the claim, fix a member $\tau_{0}$ of the chain, and an arbitrary integer $n$. Then there is some $\sigma_{i}, i>n$, extending $\tau_{0}$, and a member $\tau_{1}$ of the chain with length greater than $\left|\sigma_{i}\right|$. Let $\sigma_{j}$ be an extension of $\tau_{1}$, for some $j>i$. Then if $\sigma_{i}$ is not on the chain, there must be some $k$ in the interval $(i, j)$ such that $\sigma_{k}$ is an initial segment of $\tau_{1}$. Since $n$ was arbitrary, the claim follows.

Now extend $\leq_{t}$ to a wellordering $\leq_{T}$ of $T$, and let $\gamma$ be the length of $\leq_{T}$. Let $h: \omega_{1} \rightarrow T$ be a bijection, and define $g: \omega_{1} \rightarrow \omega_{1}$ by letting $g(\alpha)$ be the ordertype of $\leq_{T}$ restricted to $h[\alpha]$. Then $g$ is a canonical function for $\gamma$. Furthermore, for a club $C^{\prime} \subset C$ of $\alpha<\omega_{1}, T \uparrow(\omega \times \alpha)=h[\alpha]$. For these $\alpha, g(\alpha)$ is greater than the ordertype of every wellordering in the projection of $T \uparrow(\omega \times \alpha)$, and thus greater than $f(\alpha)$.

5.6 Remark. Note that Suslin Bounding for $\Pi_{1}^{1}$ sets and Suslin Bounding for $\prod_{\sim}^{1}$ sets are identical. 
Instead of working directly with the definition of universal Baireness, we will consider the equivalent (in the presence of large cardinals) form given by Theorem [5.8. Given a set $X$, we let $m(X)$ denote the set of countably complete ultrafilters on $X$. Recall that a sequence of measures $\left\langle\mu_{i}: i<\omega\right\rangle$ such that each $\mu_{i}$ concentrates on $\kappa^{i}$ is a countably complete tower if for any sequence $\left\langle A_{i}: i<\omega\right\rangle$ such that each $A_{i} \in \mu_{i}$ there is a sequence $z \in \kappa^{\omega}$ such that each $z \nmid i \in A_{i}$. See [7, 15] for more detail.

5.7 Definition. Suppose that $\kappa$ is a nonzero ordinal and that $T$ is a tree on $\omega \times \kappa$. Then $T$ is $\delta$-homogeneous if there is a partial function $\pi: \omega^{<\omega} \rightarrow m\left(\kappa^{<\omega}\right)$ such that

1. if $s \in \operatorname{dom}(\pi)$ then $\pi(s)$ is a $\delta$-complete measure on $\kappa^{|s|}$ and $\pi(s)\left(T_{s}\right)=1$, where $T_{s}=\left\{t \in \kappa^{|s|}:(s, t) \in T\right\}$,

2. for all $x \in \omega^{\omega}, x \in p[T]$ if and only if

(a) $\{x\lceil k: k \in \omega\} \subset \operatorname{dom}(\pi)$,

(b) $\langle\pi(x\lceil k): k \in \omega\rangle$ is a countably complete tower.

A set $A \subset \mathbb{R}$ is $\delta$-homogeneously Suslin if $A=p[T]$ for some $\delta$-homogeneous tree T. $A$ is ${ }^{\infty}$-homogeneously Suslin if it is $\delta$-homogeneously Suslin for arbitrarily large $\delta$.

Theorem 5.8. ([4]) Suppose that there is a proper class of Woodin cardinals and that $A \subset \mathbb{R}$. Then the following are equivalent.

1. A is universally Baire.

2. A is ${ }^{\infty}$-homogeneously Suslin.

We use the following fact to ensure that our forcing iteration considers all universally Baire sets.

Lemma 5.9. Let $P$ be an $(\omega, \infty)$-distributive partial order, and let $G \subset P$ be $V$ generic. Then in $V[G]$, for all $A \subset \mathbb{R}$ and all $\gamma>\left(2^{|P|}\right)^{+}, A$ is $\gamma$-homogeneously Suslin if and only if $A \in V$ and $A$ is $\gamma$-homogeneously Suslin in $V$.

Proof. This follows from the following standard facts about measures, where $V[G]$ is an extension by a forcing $P$ such that $\left(2^{|P|}\right)^{+}<\gamma \leq \kappa$.

1. For every $\gamma$-complete measure $U$ on $\kappa^{<\omega}$ in $V[G], U \cap V \in V$. (Otherwise, densely often in $P$ there is a set in $V$ whose membership in the measure is undecided; by genericity then there will be a subset of the measure of size $\leq|P|$ with empty intersection.)

2. For every $\gamma$-complete measure on $\kappa^{<\omega}$ in $V[G]$, every positive set contains a positive set in $V$. (For each $P$-name for a positive set and each condition in $P$, consider the set of sequences $p$ forces into the positive set.) 
3. Every $\gamma$-complete measure on $\kappa^{<\omega}$ in $V$ extends to one in $V[G]$. (All sets containing positive sets from the ground model.)

For the forward direction, let $T$ be a $\gamma$-homogeneous tree on $\omega \times \kappa$ in $V[G]$ such that $p[T]=A$, for some $\gamma>\left(2^{|P|}\right)^{+}$. Let $\pi: \omega^{<\omega} \rightarrow m\left(\kappa^{<\omega}\right)$ witness that $T$ is $\gamma$-homogeneous. Each $\pi(\sigma)$ extends a measure in $V$ on $\kappa^{<\omega}$, and since $P$ is $(\omega, \infty)$-distributive, the corresponding function $\pi^{\prime}$ taking each $\sigma$ to the restriction of $\pi(\sigma)$ to $V$ exists in $V$. For each $x \in \mathbb{R} \backslash p[T]$, let $\left\langle A_{k}^{x}: k<\omega\right\rangle$ be a witness to the fact that $\langle\pi(x\lceil k): k<\omega\rangle$ is not countably complete. For each $s \in \omega^{<\omega}$, let

$$
B_{s}=\bigcap\left\{A_{|s|}^{x} \mid x \in \mathbb{R} \backslash p[T] \wedge s \subset x\right\} .
$$

Since every positive set for each $\pi(x\lceil k)$ contains one from $V$, and since $P$ is $(\omega, \infty)$-distributive, we can assume by shrinking if necessary that $\left\langle B_{s} \mid s \in \omega^{<\omega}\right\rangle$ is in $V$. Now let $T^{\prime} \in V$ be the set of pairs $s, t$ such that $t \in B_{s}$. Since the measures are all $\gamma$-complete, each $B_{s}$ is positive for $\pi(s)$. Then $T^{\prime}$ is $\gamma$ homogeneous (with $\pi^{\prime}$ as a witness) with the same projection as $T$.

For the other direction, assume that $T($ on $\omega \times \kappa)$ and $\pi$ in $V$ witness that $A$ is $\gamma$-homogeneously Suslin. Extend the $\pi(\sigma)$ 's to $V[G]$-measures, inducing a function $\pi^{\prime}: \omega^{<\omega} \rightarrow m\left(\kappa^{<\omega}\right)$. Since each positive set in $V[G]$ contains one in $V$, and since no $\omega$-sequences of ordinals have been added by $P$, for each $x \in \omega^{\omega}$ the countable completeness of the corresponding tower is not changed by $P$. Since no countable sets of ordinals have been added the projection of $T$ is the same, so $T$ (along with $\pi^{\prime}$ ) witnesses in $V[G]$ that $A$ is $\gamma$-homogeneously Suslin.

Let $T$ be a tree on $\omega \times \kappa$ and let $f: \omega_{1} \rightarrow p[T]$. Our one-step forcing $R_{f, T}$ is the set of continuous increasing sequences $\left\langle x_{\alpha} \in[\kappa]^{<\omega_{1}}: \alpha \leq \beta\right\rangle$ of countable length such that for all $\alpha \leq \beta, x_{\alpha} \cap \omega_{1} \in \omega_{1}$ and $f\left(x_{\alpha} \cap \omega_{1}\right) \in p\left[T\left\lceil\left(\omega \times x_{\alpha}\right)\right]\right.$, ordered by extension.

Given a $\delta^{+}$-homogeneous set of reals, we use the measures witnessing homogeneity to suitably expand countable elementary substructures of $H(\chi)$.

Lemma 5.10. Fix $\delta \geq \omega_{1}$, and let $T$ be a $\delta^{+}$-homogeneous tree on $\omega \times \kappa$, witnessed by $\pi: \omega^{<\omega} \rightarrow m\left(\kappa^{<\omega}\right)$. Let $\chi>2^{\kappa}$ be a regular cardinal with $\leq_{\chi}$ a wellordering of $H(\chi)$, and let $X \prec\left(H(\chi), \in, \leq_{\chi}\right)$ with $T, \pi \in X$. Then for any countable $a \subset p[T]$ there exists a countable $z \subset \kappa$ such that letting $Y=$ $S k_{\left(H(\chi), \in, \leq_{\chi}\right)}(X \cup z)$ we have that

1. $X \cap \delta=Y \cap \delta$,

2. $a \subset p[T \uparrow(\omega \times(Y \cap \kappa))]$.

3. $[z]^{<\omega} \subset \bigcap\left\{D_{\kappa}^{Z}: Z \prec\left(H(\chi), \in, \leq_{\chi}\right) \wedge\{T, \pi\} \in Z \in X\right\}$.

Proof. Fix T, $\pi, X$ and $a=\left\{a_{i}: i<\omega\right\}$. By Lemma 2.1, it suffices to show that we can deal with $a_{0}$, as we can just repeat the process $\omega$ times. For each $k<\omega$, let $A_{k}=\bigcap\left(X \cap \pi\left(a_{0}\lceil k)\right)\right.$. Then by the definition of $\delta^{+}$-homogeneity (i.e., countable completeness) there exists $z \in(\kappa \backslash \delta)^{\omega}$ such that for all $k<\omega$, $\left(a_{0}\left\lceil k, z\lceil k) \in T\right.\right.$ and $z\left\lceil k \in A_{k}\right.$. Now $z$ is as desired, since by the $\delta^{+}$-completeness 
of each $\pi\left(a_{0}\lceil k)\right.$, if $k \in \omega$ and $h: \kappa^{k} \rightarrow \delta$ then $h$ is constant on a set $C_{h} \in \pi\left(a_{0}\lceil k)\right.$, and so this constant value must be an element of any elementary submodel of $\left(H(\chi), \in, \leq_{\chi}\right)$ with $h$ and $\pi$ as members. Further, if $h$ is in $X$, then $z\left\lceil k \in C_{h}\right.$, so $h(z\lceil k)$ is the corresponding constant value.

Given $Y$ as in Lemma 5.10 where $f\left(Y \cap \omega_{1}\right) \in a$, the union of any $Y$-generic for $R_{f, T}$, adjoined by its union, is a condition.

To get the consistency of Suslin Bounding from Theorem 3.2 we start from a proper class of Woodin cardinals, and let $\kappa$ be a strongly inaccessible cardinal such that every ${ }^{\infty}$-homogeneously Suslin set of reals is ${ }^{\infty}$-homogeneously Suslin in $V_{\kappa}$. The assumption of the Woodin cardinals is just to make ${ }^{\infty}$-homogeneous Suslinity equal to universal Baireness. Let $F: \omega_{1} \rightarrow \mathbb{R}$ be a wellordering of the reals. Let our bookkeeping for $P$ be such that each pair $(A, f)$, where $A$ is a universally Baire set (from the ground model) and $f: \omega_{1} \rightarrow A$ is added by some initial segment of the iteration, is associated to some $\rho$ greater than the stage at which $f$ was added, such that $A$ is the projection of a $\left|\mathcal{P}\left(\mathcal{P}\left(\lambda_{\rho}\right)\right)\right|^{+}$homogeneously Suslin tree $T \in V_{\kappa}$ on $\omega \times \kappa$ for some $\kappa \leq \lambda_{\rho+1}$. For this $\rho$, we let each $A_{\beta}^{\rho}$ be the set of countable $x \subset \lambda_{\rho+1}$ such that $F(\beta) \in p[T \uparrow(\omega \times(x \cap \kappa))]$, so that $Q_{\rho,\left(F^{-1} \circ f\right)}=R_{f, T}$. By Lemma 5.10, these sets also satisfy Conditions 1 1.3 of Theorem 3.2. This scheme then gives the following corollary.

Corollary 5.11. Suppose that there is a proper class of Woodin cardinals and let $\kappa$ strongly inaccessible be such that every ${ }^{\infty}$-homogeneously Suslin set of

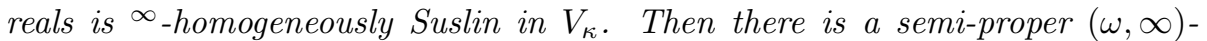

distributive forcing of size $\kappa$ in whose extension the Continuum Hypothesis and Suslin Bounding hold.

\section{References}

[1] O. Deiser, H.-D. Donder, Canonical functions, non-regular ultrafilters and Ulam's problem on $\omega_{1}$, J. Symbolic Logic 68 (2003), no. 3, 713-739

[2] H.-D. Donder, U. Fuchs, Revised Countable Support Iterations, Handbook of Set Theory, to appear

[3] Q. Feng, T. Jech, Projective Stationary sets, and Strong Reflection Principles, J. London Math. Soc. (2) 58 (1998), 271-283

[4] Q. Feng, M. Magidor, W.H. Woodin, Universally Baire sets of reals, Set theory of the continuum (Berkeley, CA, 1989), 203-242, Math. Sci. Res. Inst. Publ., 26, Springer, New York, 1992

[5] T. Jech, Set Theory, 2nd edition, Perspectives in Mathematical Logic. Springer-Verlag, Berlin, 1997

[6] P. Larson, The Canonical Function Game, in preparation 
[7] D.A. Martin, J.R. Steel, A proof of projective determinacy, J. Amer. Math. Soc. 2 (1989), 71-125

[8] T. Miyamoto, A Limit Stage Construction for Iterating Semiproper Preorders, in preparation

[9] T. Miyamoto, Preserving a Stronger Form of Semiproperness under the Simple Iteration, in preparation

[10] Y. Moschovakis, Descriptive Set Theory, Studies in Logic and the Foundations of Mathematics, 100, North-Holland Publishing Co., Amsterdam-New York, 1980

[11] S. Shelah, A more general iterable condition ensuring $\aleph_{1}$ is not collapsed, II, Publication number 311, in preparation

[12] S. Shelah, Proper and improper forcing, Perspectives in Mathematical Logic, Springer-Verlag, Berlin, 1998

[13] S. Shelah, More on Weak Diamond, Publication number 638 Available at http://front.math.ucdavis.edu/math.LO/9807180

[14] S. Shelah, NNR revisited, Publication number 656 Available at http://front.math.ucdavis.edu/math.LO/0003115

[15] W.H. Woodin, The axiom of determinacy, forcing axioms, and the nonstationary ideal, DeGruyter Series in Logic and Its Applications, vol. 1, 1999

Department of Mathematics and Statistics

Miami University

Oxford, Ohio 45056

USA

larsonpb@muohio.edu

Institute of Mathematics

Department of Mathematics

Hebrew University

Rutgers University

91904 Jerusalem

New Brunswick, NJ 08903

Israel

USA

shelah@math.huji.ac.il 